\title{
Assessing Traumatic Event Exposure: General Issues and Preliminary Findings for the Stressful Life Events Screening Questionnaire
}

\author{
Lisa A. Goodman, ${ }^{1,5}$ Carole Corcoran, ${ }^{2}$ Kiban Turner, ${ }^{3}$ Nicole Yuan, ${ }^{4}$ \\ and Bonnie L. Green ${ }^{4}$
}

This article reviews the psychometric properties of the Stressful Life Events Screening Questionnaire (SLESQ), a recently developed trauma history screening measure, and discusses the complexities involved in assessing trauma exposure. There are relatively few general measures of exposure to a variety of types of traumatic events, and most of those that exist have not been subjected to rigorous psychometric evaluation. The SLESQ showed good test-retest reliability, with a median kappa of .73, adequate convergent validity (with a lengthier interview) with a median kappa of .64, and good discrimination between Criterion $A$ and non-Criterion $A$ events. The discussion addresses some of the challenges of assessing traumatic event exposure along the dimensions of defining traumatic events, assessment methodologies, reporting consistency, and incident validation.

KEY WORDS: traumatic events measure; psychometrics; trauma history assessment; trauma exposure.

Over the last 15 years, a variety of psychometrically sound measures of posttraumatic stress disorder have been developed (PTSD; American Psychiatric Association [APA], 1994). Similarities among measures such as the Penn Inventory (Hammarburg, 1992), the PTSD Symptom Scale (Foa, Riggs, Dancu, \& Rothbaum, 1993) and the Civilian Mississippi Scale (Nor-

${ }^{1}$ Psychology Department, University of Maryland, College Park, College Park, Maryland 20742. ${ }^{2}$ Psychology Department, Mary Washington College, Fredericksburg, Virginia 22401.

${ }^{3}$ Psychology Department, Duke University, Durham, North Carolina 27706.

${ }^{4}$ Department of Psychiatry, Georgetown University, Washington DC 20057.

${ }^{5}$ To whom correspondence should be addressed (e-mail: lgoodman@bss3.umd.edu). 
ris \& Perilla, 1996; Vreven, Gudanowski, King, \& King, 1995) indicate a growing consensus on how to measure this construct. However, while such measures address the $B$ (reexperiencing), $C$ (avoidance-numbing), and D (arousal) symptoms of the disorder, they tend not to address the A1 (exposure) criterion independently. This failure to include exposure assessment in measures of PTSD is, in part, due to the way in which the trauma field has developed. Research studies have tended to focus on sequelae of traumatic events within specific populations of survivors (e.g., Vietnam veterans, rape survivors, disaster survivors), and trauma researchers have tended to develop separate and stand-alone exposure measures for discrete types of traumatic events.

However, recent research suggests that it is common for people to experience multiple traumatic events in the course of their lives (e.g., Kessler, Sonnega, Bromet, Hughes, \& Nelson, 1995; Norris, 1992), that prior exposure to traumatic events may affect subjects' responses to a later event (e.g., Resnick, Kilpatrick, Dansky, Saunders, \& Best, 1993), and that the effects of traumatic experiences may be cumulative (Follette, Polusny, Bechtle, \& Naugle, 1996; Goodman, Dutton, \& Harris, 1997). Thus, it is imperative that researchers develop psychometrically sound measures of lifetime exposure to a variety of traumatic events, even for studies which focus on a target traumatic event.

This paper describes the challenges of assessing traumatic event exposure. These challenges arise across a number of dimensions, including definitional concerns, assessment methodologies, reporting consistency, and incident validation. We begin with a brief review of existing traumatic event exposure measures and then describe the Stressful Life Events Screening Questionnaire (SLESQ), a recently developed comprehensive screening measure of traumatic event exposure designed for use with non-treatment seeking (community) samples (see Appendix). Next, we present preliminary information on the specificity, reliability, and validity of the SLESQ. Finally, we discuss some of the complex issues that arose as we developed the measure and interpreted the results of its psychometric evaluation.

\section{Existing Measures}

A number of recently published measures aim to assess lifespan traumatic event exposure (for reviews, see Krinsley \& Weathers, 1995; Norris \& Riad, 1997; Resnick, Falsetti, Kilpatrick, \& Freedy, 1996). These scales differ with regard to their goals, whether they are administered in self-report or interview format, how narrowly they define traumatic events, and 
the extent to which objective and subjective descriptive information is obtained.

With regard to breadth of definition, the Traumatic Stress Schedule (TSS; Norris, 1990), is a brief screening interview that defines the event domain as encompassing eight "violent encounters with nature, technology, or humankind"' (p. 1706). The Traumatic Events Questionnaire (TEQ; Vrana \& Lauterbach, 1994), also self-report, elicits information on 11 events and includes several open-ended probes for "other" stressful experiences. Similarly the self-report Traumatic Life Events Questionnaire (TLEQ; Kubany, 1995) assesses the occurrence of 17 potentially life-threatening events, and the self-report Trauma History Questionnaire (THQ; Green, 1996) samples a still broader population of relevant events with 23 specific scale items. In contrast, the Potential Stressful Events Interview (PSEI; Resnick, Falsetti, Kilptrick, \& Freedy, 1996), a structured in-depth interview used in the DSM-IV field trials, assesses both high and low magnitude stressors, including 13 events that potentially involve life threat. The Evaluation of Lifetime Stressors interview (ELS; Krinsley \& Weathers, 1995) offers the most comprehensive assessment of highly stressful experiences and categorizes these events as either subthreshold stressors, potentially traumatic events, or traumatic events.

In addition to employing varying definitional boundaries for the inclusion of traumatic events, these measures differ greatly in the follow-up details elicited, the time necessary for administration, the type of response formats, and the degree of available psychometric support. Adequate reliability has been established for the TSS (Norris, 1990; Norris \& Perilla, 1996), the THQ (Green, 1996), and the TEQ (Lauterbach \& Vrana, 1996) with regard to overall number of events reported across two test administrations. However, only three measures have undergone reliability testing for specific items. One of the authors of this paper (BLG) found that in a small sample of college students $(n=25)$, test re-test correlation coefficients over a 2-to 3-month period for the 23 specific items of the THQ ranged from .47 to 1.00 , with a median of .64 . With regard to the ELS, Krinsley, Gallagher, and Weathers ( $K$. Krinsley, personal communication, October 11,1996$)$ found that in their preliminary sample of 40 Vietnam veterans test-retest kappas ranged from .31 to 1.00 , with a median kappa of .67 for adult traumatic events and .48 for childhood traumatic events. No psychometric data are currently available for the PSEI or the TLQ.

What psychometric evaluations of these omnibus traumatic event exposure measures have made clear is that gathering data about past traumatic exposure is not necessarily as straightforward as collecting data on other more clearcut characteristics, such as demographics. Rather, assessment of traumatic event exposure is a complex measurement issue, involv- 
ing issues of definition, assessment methodology, consistency of reporting, and validity of reports. Appropriate psychometric validation is therefore necessary.

The SLESQ was developed as a general traumatic event screening questionnaire for use in non-treatment seeking samples. At the time we began this project, only one comprehensive screening measure was available in the literature (Norris, 1992). As noted, a number of published and unpublished instruments are now in development, although few have reported psychometric characteristics. In the discussion, we address the relative advantages of the SLESQ and the specific uses for which it is best suited.

\section{Method}

\section{Measure Development: The SLESQ}

The SLESQ is a 13-item self-report screening measure designed to assess lifetime exposure to a variety of traumatic events. The measure was developed in the context of a research study that required a comprehensive self-report traumatic event exposure screening questionnaire to be administered to a large pool of respondents, a subset of whom would then be followed-up with face-to-face interviews. The purpose of the measure is to identify as quickly and efficiently as possible all traumatic events experienced by respondents, while avoiding subthreshold events that would not be conceptualized as "traumatic," using the threshold set in Criterion A1 of the PTSD diagnosis (DSM-IV, APA, 1994). It does not address Criterion A2 (subjective reaction to the event.) Consistent with Criterion A1, we defined a traumatic event as one that "involves actual or threatened death or serious injury, or a threat to the physical integrity of self or others" (p. 427). Using that definition, we developed a pool of 11 specific event categories and two general categories for events that might meet Criterion A but that did not fall into the more specific categories. These categories were based on: (a) a review of a number of available traumatic event exposure questionnaires that target either specific events (e.g., Koss \& Oros, 1982; Russell, 1986; Straus, 1989) or sample a range of event types (e.g., Green, 1996; Norris, 1990; Resnick et al., 1993); and (b) subsequent pilot testing of our measure. Throughout the process of measure development, we adhered to fairly stringent definitions of traumatic events (see Appendix A for a copy of the SLESQ). Although the events covered by the SLESQ overlap significantly with many of the measures described above, the SLESQ places less emphasis than other screening measures (e.g., the TSS; 
Norris, 1990; and the TEQ, Vrana \& Lauterbach, 1994) on disasters and more emphasis on behaviorally specific assessment of traumatic events of an interpersonal nature. Because the SLESQ was designed to be relatively brief, it does not assess traumatic event exposure in as much detail as an in-depth interview such as the PSEI (Resnick et al., 1996) or the ELS (Krinsley \& Weathers, 1995). It does, however, elicit information about some important details (e.g., level of force used and injury received) for each endorsed event.

We conducted two pilot projects in order to refine the items contained in the initial measure. For the first pilot, designed to assess the extent to which SLESQ questions were behaviorally concrete and specific, we administered the initial version of the SLESQ to 265 college women from four area campuses. We then evaluated respondents' responses to the openended questions to assess whether the information provided was clear enough to categorize the events endorsed as traumatic or not traumatic according to our definitions and whether there were events reported in the "other" category that we wished to include among the standard items. This pilot resulted in the addition of several questions eliciting more details about specific events, but no new event categories.

The second pilot project was designed to provide an initial assessment of the reliability and validity of the SLESQ. The revised (on the basis of pilot 1 results) instrument was administered to 60 new respondents, including both male and female college students. Two weeks later, 30 of these respondents completed the self-report questionnaire again, and 30 were interviewed in-person about the same domain of events using a structured interview. Although test-retest reliability was generally adequate, we found that the interview protocol elicited a number of traumatic events that were not picked up in the screening measure, particularly in the area of physical assault. This category may have been insufficiently covered in the initial version of the measure because it was first piloted with women only. We therefore modified the screening items to address this gap.

In the final version of the SLESQ, participants are asked whether they have experienced each of 11 events and two "catch-all" experiences. If they answer affirmatively, they are asked to provide additional information (depending on the item), including the following: age (of self and perpetrator), a brief description of the event, extent of injuries, nature of force involved, whether there was a perceived life threat, relationship to perpetrator, frequency of occurrence, and duration of ongoing traumatic experiences. This descriptive information is particularly important for the catch-all questions because it enables researchers to learn some of the details of the event so that they can determine whether it fits with a Criterion A1 definition rather than relying on respondents' subjective determinations. Further, the use of 
open-ended questions enables some flexibility in defining threshold criteria for a traumatic event, depending on the purpose of the research. Responses to the open-ended questions could be coded quite easily.

\section{The Present Study}

The specificity, reliability, and validity of the final version of the SLESQ were assessed using a sample of college students. We chose this population for convenience and because the SLESQ was initially developed for use in this population. In order to establish the specificity of the measure, i.e., its ability to pick up primarily Criterion A events rather than subthreshold events, we evaluated each endorsed item of a systematically selected subsample of screening questionnaires (every third screening measure with a random start; $n=46$ ) against a conservative definition of Criterion A traumatic events developed for each category. Decisions about traumatic event thresholds were made by consensus of the first three authors.

To meet our criteria, events had to be life-threatening, to involve a significant level of violence and/or assault to bodily integrity, and to have occurred to the respondent or to an extremely close other. For example, if a respondent affirmed that a "very close friend" committed suicide, we examined the qualitative descriptions to determine how the respondent conceptualized 'very close friend." If this was someone with whom the respondent had attended elementary school but with whom they have had little contact since, we categorized this event as "subthreshold." With regard to physical assault, we "counted" as traumatic repeated beatings with a belt or other object, actions more commonly (though not always) understood to be abusive, but not spanking or sibling fights. With respect to life-threatening illnesses, we "counted" cancer, an illness that is usually viewed as life-threatening, but not pneumonia, an illness that is rarely viewed as life-threatening.

We recognize that these cut-offs are somewhat arbitrary. For example, decisions regarding what kinds of "physical punishment" cross the line into traumatic or abusive events are influenced by cultural and personal values. However, the equally problematic alternative was to accept any positive response, without imposing standard criteria. Whether a researcher leans towards objective or subjective definitions of events may depend on the purpose of the research. Since the SLESQ was designed to maximize reporting of Criterion $A$ events and to minimize reporting of non-Criterion A events (no matter how awful), we decided to develop conservative cut-offs and to make them explicit so that others may inter- 
pret our conclusions accordingly. Some empirical support for the meaningfulness of our criteria comes from a preliminary analysis of data from the larger study of which this is a part. Using the 850 screening measures collected so far, we compared college women who simply checked off "yes" to the traumatic bereavement response (regardless of the identity of the person who died) with college women who reported no traumatic exposure. Scores of the two groups did not differ on any subscale of the Traumatic Symptom Inventory (TSI; Briere, Elliott, Harris \& Cotman, 1995), a measure of a broad range of post-traumatic symptoms. However, when we compared the control respondents to respondents whose bereavement involved a person with whom they were living or having frequent contact, we did find significant differences on several subscales of the TSI, with traumatically bereaved respondents scoring higher on anxious arousal, irritability, depression, defensive avoidance, dissociation, and intrusive experiences, $t$ 's $=1.98-3.53, d f$ 's $=186-187, p$ 's $<.05-.01$. When data collection is finished, we will be able to report this kind of information for each separate type of event.

Second, in order to establish concurrent validity, we compared prevalence rates obtained using the SLESQ to those obtained in other studies examining the prevalence of traumatic events. For this and all subsequent analyses, we used all responses, whether or not they met the conservative cut-off established for determining specificity. Third, we used test-retest reliability to establish the questionnaire's stability within each event category and overall. Finally, to establish sensitivity and convergent validity, we assessed whether or not a subset of respondents provided the same responses to a more detailed in-person interview (considered the gold standard in traumatic event assessment) 2 weeks later as they did to the questionnaire. This interview adapted questions from three established interviews: Russell's (1986) semi-structured sexual abuse interview; the high magnitude stressors portion of the PSEI (Resnick et al., 1996), the trauma history interview used in the DSM-IV field trials; and the Conflict Tactics Scale (Straus, 1989), a measure of physical assault experiences which can be administered in interview or self-report form.

Sample and procedure. Respondents included 202 male and female summer school students at a large eastern university, recruited through their psychology classes. Approximately $75 \%$ of those who were informed about the study chose to participate. They were told that we were conducting a study to learn whether a survey that we had developed reliably measures stressful events students may have experienced in the course of their lives. Respondents were told that the testing would take place on two occasions, 2 weeks apart, but they were not told that some of them would be completing the same questionnaire twice. After signing informed con- 
sents, those who agreed to participate were asked at the end of the class period to complete a background information form as well as the SLESQ and then to return 2 weeks later to complete a second questionnaire or participate in an interview. Of the original 202 respondents, $140(69 \%)$ returned for the follow-up. These respondents were randomly assigned either to a second group administration of the questionnaire $(n=66)$ or a 30 to 40-min interview covering the same areas in more detail with a trained clinical interviewer $(n=74)$. Respondents who returned were paid $\$ 5.00$ at the completion of the session.

\section{Results}

\section{Sample Characteristics}

There were no significant differences in the age, gender, ethnicity, or mean number of events reported between the 62 respondents who failed to return after the first test administration and the 140 respondents who completed both parts of the study. There were also no significant group differences in the number of respondents who reported each category of event, with the exception of "life-threatening illness" $t(193.7)=2.65, p<$ .01 (with correction for unequal variances), and "other injury or life threat" $t(187)=2.16, p<.05$ (with correction for unequal variances). In both cases, respondents who failed to return reported fewer events in these categories. The sample was ethnically diverse: $59 \%$ were Caucasian, $17 \%$ were African-American, $9 \%$ were Asian-American, $7 \%$ were Latino, and the remaining $8 \%$ checked "Other."

Sixty six percent of the sample were women (mean age $=22.3$ ), and $34 \%$ were men (mean age $=23.6$ ). At least one traumatic event was reported by $72 \%$ of these respondents, and the mean number of events reported was $1.83(S D=1.96)$. Since there were no significant differences in the total number of events reported by women versus men at either test administration, their data were combined for all subsequent analyses except prevalence reporting.

\section{Specificity}

Respondents endorsed a total of 81 events in the 46 systematically selected screening questionnaires. We rated 69 of these events (85\%) as meeting our severity threshold for a Criterion A event. The most commonly 
reported subthreshold items included peer fights, spanking by parents, and non-life-threatening illnesses such as mononucleosis.

\section{Concurrent Validity: A Comparison of Prevalence Rates}

Prevalence rates for the 13 SLESQ items are presented by gender in Table 1 . Child and adult physical abuse/assault, sudden bereavement, and life-threatening accidents were the most commonly experienced events. As expected, types of events experienced varied by gender. Women were significantly more likely than men to have been molested, $t(138)=2.03, p<$ .01 , and to have experienced attempted sexual assault, $t(138)=2.45, p<$ .01 . Men were significantly more likely than women to have experienced adult physical assault, $t(138)=1.97, p<.05$, and other serious injury or life threat, $t(138)=2.00, p<.05$.

Wording of items, behavioral specificity of questions, and thresholds/definitions for trauma differ across trauma history measures, making prevalence comparisons across studies difficult. However, where possible, prevalence rates for the events listed in the SLESQ were compared to rates found in prevalence studies using two large probability samples (Kessler et al., 1995 [national]; Norris, 1992 [regional]). As Table 2 shows, with the exceptions of robbery, traumatic bereavement, and witnessing death, the prevalence rates for specific events in our study were fairly consistent with

Table 1. Prevalence of Traumatic Events Experience by Gender

\begin{tabular}{|c|c|c|c|}
\hline Event & $\begin{array}{c}\text { Total } \\
N=140 \\
\%\end{array}$ & $\begin{array}{c}\text { Women } \\
n=93 \\
\%\end{array}$ & $\begin{array}{c}\text { Men } \\
n=47 \\
\%\end{array}$ \\
\hline Life-threatening illness & 13 & 9 & 21 \\
\hline Life-treatening accident & 16 & 14 & 21 \\
\hline Robbery/mugging & 6 & 5 & 9 \\
\hline Traumatic bereavement & 19 & 22 & 15 \\
\hline Sexual assault (penetration) & 11 & 13 & 6 \\
\hline Attempted sexual assault ${ }^{\alpha}$ & 8 & 11 & 2 \\
\hline Molestation $^{a}$ & 14 & 17 & 6 \\
\hline Child physical assault/abuse & 22 & 18 & 30 \\
\hline Adult physical assault/abuse ${ }^{a}$ & 18 & 13 & 28 \\
\hline Threatening with weapon & 6 & 7 & 4 \\
\hline Witnessed death/assault & 12 & 9 & 19 \\
\hline Other life threat & 11 & 6 & 19 \\
\hline Other horrifying event & 13 & 13 & 13 \\
\hline Any trauma (excluding "other") & 66 & 67 & 64 \\
\hline Any trauma (including "other") & 72 & 71 & 75 \\
\hline
\end{tabular}

${ }^{a}$ Women and men differed significantly $(p<.05)$ on prevalence. 
Table 2. Comparison of Prevalence Rates

\begin{tabular}{|c|c|c|c|}
\hline Event & $\begin{array}{c}\text { SLESQ } \\
\begin{array}{c}N=140 \\
\%\end{array}\end{array}$ & $\begin{array}{c}\text { Kessler et al., } 1995 \\
N=5877 \\
\%\end{array}$ & $\begin{array}{c}\text { Norris, } 1992 \\
N=1000 \\
\%\end{array}$ \\
\hline Life-threatening accident & 16 & 19 & 23 \\
\hline Robbery/mugging & 6 & - & 25 \\
\hline Traumatic bereavement & 19 & - & 30 \\
\hline Sexual assault (penetration) & 11 & 5 & 4 \\
\hline Molestation & 14 & 8 & - \\
\hline Child physical assault/abuse & 22 & 4 & - \\
\hline Adult physical assault/abuse & 18 & 9 & - \\
\hline Both child/adult physical A/A & - & - & 15 \\
\hline Threatened with weapon & 6 & 13 & - \\
\hline Witnessed death/assault & 12 & 25 & - \\
\hline Other horrifying/terrible event & 13 & 2 & - \\
\hline Any trauma & 72 & 56 & 69 \\
\hline
\end{tabular}

those reported by either Kessler or Norris. In addition, the prevalence rate for completed rape reported by Resnick and colleagues (1993) for women in a large representative community sample $(N=4,008)$ was quite similar $(12.65 \%)$ to our obtained prevalence rate of $13 \%$ for sexual assault with penetration (see Table 1).

\section{Test-retest Reliability: Screen-Screen Condition}

Over a 2-week test-retest interval, the correlation between the number of events reported at time 1 and the number reported at time 2 was 89 . As shown in Table 3, kappas for the occurrence of specific events ranged from .31 for attempted sexual assault to 1.00 for robbery or mugging (median $k=.73$ ). Not surprisingly, the general ("other") questions had among the lowest kappas (.25 and .40) Four items fell below a kappa of .60: attempted rape, witness to a traumatic event, other serious injury or life threatening situation such as military combat or living in a war zone, and "other frightening or horrifying event."

Overall, there were no significant differences in the proportion of respondents who reported an event the first time only versus the second time only. That is, 33\% of participants reported an event during the first administration that was omitted on the second administration, and $30 \%$ of participants reported a new event during the retest. 
Table 3. Reliability \& Validity of SLESQ

\begin{tabular}{lcc}
\hline \multicolumn{1}{c}{ Event } & $\begin{array}{c}\text { Reliability } \\
\text { (Test-Retest) } \\
k\end{array}$ & $\begin{array}{c}\text { Validity } \\
\text { (Test-Interview) }\end{array}$ \\
\hline Life-threatening iliness & .81 & $k$ \\
Life threatening accident & .94 & .90 \\
Robbery/mugging & 1.00 & .70 \\
Traumatic bereavement & .88 & .58 \\
Sexual assault (penetration) & .63 & .77 \\
Attempted sexual assault & .31 & .89 \\
Molestation & .93 & .64 \\
Child physical assault/abuse & .88 & .72 \\
Adult physical assault/abuse & .67 & .45 \\
Threatened with weapon & .73 & .47 \\
Witnessed death/assault & .57 & .46 \\
Other life threat & .25 & .26 \\
Other horrifying event & .40 & .88 \\
Any sexual assault & .74 & .08 \\
Number of traumas & $r=.89$ & .92 \\
\hline OSexual assault and attmpted & .77 \\
\hline
\end{tabular}

${ }^{a}$ Sexual assault and attempted sexual assault items combined.

\section{Sensitivity and Convergent Validity: Screen-Interview Condition}

For the screen-interview condition, the correlation between total number of events reported at time 1 (screen) and time 2 (interview) was .77 . Kappas for the occurrence of specific events (Table 3) ranged from .26 for witnessed death/assault to .90 for life threatening illness (median $k=.64$ ). Kappas for the two "other" categories showed a wide range, from .08 for other horrifying event to .88 for other life threat. Six items fell below a kappa of .60: robbery or mugging, child physical assault/abuse, adult physical assault/abuse, being threatened with a weapon, witnessing a traumatic event, and "other frightening or horrifying experience." For all six of these low kappa items, the differences in reporting across conditions was in the direction of increased reporting in the interview.

Indeed, in contrast to the screen-screen condition, many more participants reported an additional event in the interview (54\%) compared to those who omitted an event at follow-up (30\%). Thus, although a time by method ANOVA indicated a significant time main effect, $F(1,138)=25.35$, $p<.01$, this effect was qualified by an interaction between time and method, $F(1,138)=17.14, p<.01$, such that increased reporting of events occurred only for those respondents interviewed at the second administration ( $M=1.89$ for time $1, M=2.93$ for time 2 ). 
In order to explore whether the increased reporting at the time 2 interview (and subsequent low kappas on particular items) was due to the interview's relatively greater sensitivity to detect exposure to Criterion $\mathrm{A}$ events or to its elicitation of more subthreshold events, we used the eventevaluation procedure described above with respect to specificity to determine whether the additional events reported in the course of an interview met the Criterion A standard. Further, in order to determine whether differential reporting was due to timing (second administration) vs. method (interview), we also evaluated all additional events reported at the time 2 screening but not reported at the time 1 screening. Using this method, we rated $46(63 \%)$ of the additional events reported in the interview condition as subthreshold, compared to only 10 additional events (29\%) reported in the time 2 screening condition.

\section{Discussion}

Before considering the implications of our results, it is important to note the limitations of the data. First, the generalizability of these results is constrained by virtue of the fact that we evaluated the SLESQ using a relatively small sample of college students. Thus, its psychometric characteristics in other populations is yet to be determined. Indeed, this questionnaire may be less useful for populations with low education or reading difficulties. For example, some of the items list a number of different possible perpetrators. While we included a comprehensive list in order to trigger reporting of the widest possible range of events, it is possible that a few of the questions may be difficult for respondents with reading or comprehension difficulties. We plan to investigate this issue systematically. Without specific data on this point, however, we would recommend that the questionnaire be administered in interview format for less educated populations.

Second, this study addressed only preliminary psychometric characteristics of the measure. We did not seek external corroboration of events to establish the validity of the SLESQ. Also, we did not investigate the degree to which reliability could be established for reports of details of each event, such as age, perpetrator, and severity of injury, or how endorsement of particular items was related to symptoms. A study is now underway to assess this latter question.

Third, the SLESQ does not cover several types of events that would certainly meet Criterion A standards. These include torture, incarceration as a prisoner of war, terrorist attack, natural or manmade disasters, car accidents, or residential fires. With regard to the first three, because their 
occurrence is relatively rare in general community or college student samples, typically they are not included in instruments for these kinds of samples (e.g., Kessler et al., 1995; Norris, 1992). With regard to disasters, our original measure did contain a disaster item, but it picked up many false positives, thus threatening the specificity of the measure. Similarly, we did not include questions about car accidents or residential fires specifically as these could produce a high proportion of false positives. Instead, we asked about life threatening accidents generally, and then followed up with questions about level of injury. It is our hope that the general questions, one of which mentions combat experience specifically, will catch some of these events without pulling for low-magnitude events such as scary storms.

Fourth, in developing item wording, we established our own relatively conservative definitions of trauma, definitions that may or may not be in accordance with those of other researchers interested in using the SLESQ. On the other hand, despite these limitations, this study represents one of the first attempts to investigate a variety of psychometric properties of a comprehensive trauma screening measure.

The SLESQ was designed to pick up Criterion A events usually associated with PTSD and to minimize reporting of subthreshold events. As our specificity assessment demonstrated, we were largely successful in this endeavor. As we had hoped, $85 \%$ of the items endorsed in the first screening met our specific definitions of events. This is a distinct advantage of the instrument for studies evaluating psychological outcomes following Criterion $\mathrm{A}$ event exposure.

It is important to note, however, that even within the range of events that could be conceptualized as traumatic, different researchers may establish different thresholds. With regard to some events (e.g., surviving a plane crash, being shot, being raped at gunpoint) there would likely be easy agreement that the event met Criterion A for PTSD. Not every event is so clear-cut. How often or how hard does a parent have to hit a child for it to be considered a traumatic event or abuse? How is it determined whether one's life was in danger during a car crash? The alternative to establishing cut-offs is to leave the thresholds up to each individual, a procedure which also has its strengths and weaknesses. In developing the SLESQ, we wanted to maximize specificity. Therefore, we would recommend the measure to researchers interested in conservative definitions of Criterion $\mathrm{A}$ events but must caution that the wording of questions makes it less useful for those with more liberal definitions. The specific probes that follow each major question do provide some room for researchers to establish their own definitions. For example, they can choose the level of force or injury required or whether or not to include peer and sibling fights/assaults. 
With regard to concurrent validity, the prevalence rates obtained by the SLESQ were, for the most part, consistent with, or higher than, those found in several large prevalence studies, suggesting that we have not greatly compromised sensitivity. A likely explanation for the higher prevalence rates we obtained for physical and sexual assault items relative to those obtained by Kessler and colleagues (1995) or Norris (1992) may be that our questions avoided loaded terms such as rape (unlike Kessler's) and were extremely specific and concrete (unlike Norris'). Our sexual assault questions included probes about a range of potential perpetrators, specific sexual acts, and situations such as being asleep or drugged. Since the use of nonloaded terms, behaviorally specific items, and comprehensive questioning have been shown to have a profound influence on reporting rates, particularly in the area of sexual assault (Resnick et al., 1996), it is not surprising that our prevalence rates were higher than those elicited with Kessler's more loaded question or Norris' more general question. Our sexual assault prevalence rates are consistent with those reported in a national probability sample of college students (15\% for women, $4 \%$ for men), all of whom were given the Sexual Experiences Survey (Koss, Gidycz, \& Wisniewski, 1987), a detailed measure of sexual assault that uses behavioral definitions and avoids labels.

In several cases, including robbery, traumatic bereavement, and witnessing death, our rates are notably lower than those obtained by Norris or Kessler. It is not possible to determine whether the differences are due to wording, methodology, or sample characteristics such as age, social class, or ethnicity. Differential definitions of traumatic events (discussed above in relation to specificity) likely also contribute to differences among instruments and, consequently, differential reporting of prevalence rates for various events.

The SLESQ appears to have very good test-retest reliability, with an overall correlation of .89 between number of events reported at the time 1 versus time 2 screening. This figure is similar to the .88 and .91 test-retest correlations reported for the TSS (Norris \& Perilla, 1996) and the TEQ (Lauterbach \& Vrana, 1996), respectively. Moreover, with the exception of unpublished data on the THQ and the ELS, the SLESQ is the first measure to be evaluated for reliability across individual events. The median kappa for reporting on specific events across administrations was .73 , similar to the .64 figure reported for the THQ (Green, 1996) and the .67 figure reported for the ELS with regard to adult trauma (K. Krinsley, personal communication, October 16, 1996).

Only four items fell below a test-retest kappa of .60. As with the THQ, three of the less reliable items were either indirect forms of exposure (e.g., "witnessing") or "catch-all" events (e.g., "other serious injury or life threat- 
ening situation" and "other frightening or horrifying event"). The fourth item, attempted rape, required respondents to make a judgment about their own and their perpetrators' frames of mind. Since attempted rape, by definition, is not completed, respondents must decide whether the person actually intended to rape them. Those questions that were purposely open-ended, as well as those that were more open to interpretation, such as the attempted rape question, were least likely to be reliably answered, possibly because of the inherent ambiguity involved.

Although the SLESQ demonstrated good overall test-retest reliability, it is important to note that the test-retest reliability kappas for individual items were far from perfect. While we constructed the SLESQ to be as specific and concrete as possible and to contain multiple memory cues, respondents nevertheless failed to report events as serious as a rape until the second administration. While some of the inconsistent reporting may have been due to the wording of our questions, inconsistent reporting is probably also a more general phenomenon. Several researchers have noted that reporting on the occurrence of a traumatic event is not as straightforward as reporting on where one lives or one's family composition (e.g., Krinsley, Gallagher, \& Weathers, 1996).

It is unlikely that factors such as amnesia or dissociation played a significant role in the inconsistent reporting on the SLESQ given the short time-frame ( 2 weeks) between the first and second administrations. Nevertheless, these phenomena may play a role in inconsistent reporting generally, since a growing number of studies have demonstrated that individuals can lose and then recover memories of past trauma (Briere \& Conte, 1993; Williams, 1995), and amnesia for aspects of an event is a PTSD criterion (APA, 1994).

Other factors might also be implicated in the inconsistencies in trauma reporting. It may be that the questions asked during the initial administration of the measure caused respondents to think about experiences that they had not thought about for a long time, leading to increased reporting on the second administration. Alternatively, happenstance occurrences during the intervening 2 weeks, including having a conversation with someone or being in a place reminiscent of the original event, may have triggered recall or even recast the perceived nature of an experience. However, these are not complete explanations since a similar proportion of events $(30 \%)$ were reported only on the first screen administration. Another explanation for potential inconsistencies is that a respondent's state-of-mind may change across administrations, leading to changes in ability or motivation to retrieve remote memories at any given time or shifts in appraisal of a specific event (e.g., whether a rape had been attempted, whether a situation was life-threatening) which could then influence reporting. It is likely that 
these explanations apply to trauma history measures generally and not just to our instrument.

Overall, the SLESQ demonstrated good convergent reliability, with a correlation of .77 between number of events reported on the screening measure and number of events reported in an interview 2 weeks later. Of the six items with kappas below .60, two were "catch-all" categories, and the remaining four would have been much higher except for the tendency to report subthreshold events in the time 2 interview. Thus, most $(64 \%)$ of the additional events reported in the interviews were actually events that we did not define as potential Criterion $A$ events and did not intend to pick up on the screen, including peer and sibling fights and spanking by parents.

Respondents may be reporting additional events in the interview because they believe that as long as an interviewer is filtering their experience, they may as well mention anything that comes to mind and let the interviewers decide how to define the experiences. Alternatively, it may be more gratifying to describe events to a sympathetic interviewer than to check off an item on a questionnaire. Finally, the extensive cueing that is part of the interview process may have triggered memories of less serious or dramatic events.

\section{Uses and Further Psychometric Evaluation of the SLESQ}

In sum, the SLESQ appears to be an efficient trauma screening measure and the first to have been subjected to multiple forms of validation. Preliminary psychometric evaluation demonstrates that the SLESQ has excellent specificity and test-retest reliability and good concurrent and convergent validity. Despite these promising data, further psychometric evaluation of the SLESQ is necessary. Additional information on construct validity could be obtained by examining the relationships between SLESQ scores and specific outcome measures and by obtaining external corroboration of a subset of reported experiences. While external corroboration would be challenging, a number of investigators have begun to attempt to corroborate reports of traumatic event exposure (see, e.g., Krinsley et al., 1996). However, it seems unlikely that it would be possible to obtain evidence on all reported events. Further, there is no way to know whether some events occurred which were not reported. Thus, the SLESQ and similar instruments will, to a large extent, continue to have to rely on respondents' word regarding traumatic events. Psychometric studies of the SLESQ with a range of populations would also be useful. College students are unrepresentative of the general population in terms of developmental stage, education and socioeconomic status. The degree to which items can be read 
and understood should be assessed among respondents in clinical and community settings.

With regard to potential uses for the SLESQ, it is important to note that this measure was developed as a screening instrument. Thus, we do not see it as an alternative to more detailed measures, especially when a specific type of event is central to a particular study. In our own work, we use the instrument to screen for exposure; the screening is followed by a more detailed interview to confirm or clarify exposure information before assigning each respondent to a particular trauma category. The SLESQ might also be useful in situations where only a general accounting of exposure is needed.

With regard to the more general issues raised by this study, it seems clear that psychometric evaluation of traumatic event exposure measures, although difficult, needs to be conducted in order to advance our understanding of which types and aspects of exposure place individuals at risk for specific negative outcomes. A number of factors make this task more difficult than might be expected. As this and other studies (see, e.g., Krinsley et al., 1996) indicate, even with very consistent questions and short time periods between measure administrations, reporting of potentially traumatic events is not completely reliable. Further research needs to be conducted to ascertain whether certain types of events are more reliably reported than others. It seems clear, however, that "catch-all" items, such as those that introduce the PTSD section of the Structured Interview for DSM-III-R (Spitzer, Williams, Gibbons, \& First, 1990) pick up traumatic events less reliably than those describing a particular event, where the cues would be expected to provide a more consistent structure for remembering. Such inconsistency has clinical and research implications. For example, respondents or clients may need to be evaluated on more than one occasion to obtain a true picture of their traumatic event histories.

Even with reliable reporting, investigators may continue to disagree about how to define a "traumatic event" or a "potentially traumatic event." The DSM-IV gives some general guidelines, but these must be translated for any particular study. On the one hand, there appears to be some consensus on generic dimensions of events (Green, 1993) and on specific types of events (e.g., Kessler et al., 1995; Norris, 1992; Resnick et al., 1993) that fall within the domain of Criterion A1. On the other hand, investigators must establish their own threshold criteria (within these categories), and consensus is likely to be lower at this more specific level. The extent to which respondents' own appraisals are incorporated into definitions is also not consistent across studies. For example, who determines whether an accident or encounter was "life threatening?" While the respondent's assessment will almost certainly be a good predictor of their psychological 
response, it also merges subjective and objective aspects of stressor definitions.

In spite of these difficulties, investigators using very different methodologies have derived quite similar numbers with regard to traumatic event exposure in the general population (e.g., Kessler et al., 1995; Norris, 1992; Resnick et al., 1993). This should reassure us that the goal of developing better and more psychometrically sound measures is obtainable and worth pursuing.

\section{APPENDIX: Stressful Life Events Screening Questionnaire}

The items listed below refer to events that may have taken place at any point in your entire life, including early childhood. If an event or ongoing situation occurred more than once, please record all pertinent information about additional events on the last page of this questionnaire. Please print or write neatly.

1. Have you ever had a life-threatening illness?

$$
\text { No ___ Yes ___ If yes, at what age? }
$$

Duration of Illness (in months)

Describe specific illness

2. Were you ever in a life-threatening accident?

$$
\text { No Y Yes __ If yes, at what age? }
$$

Describe accident

Did anyone die? Who? (relationship to you)

What physical injuries did you receive?

Were you hospitalized overnight? No___ Yes

3. Was physical force or a weapon ever used against you in a robbery or mugging?
No
Yes
If yes, at what age?

How many perpetrators?

Describe physical force (e.g., restrained, shoved) or weapon used against you.

Did anyone die? Who?

What injuries did you receive?

Was your life in danger?

4. Has an immediate family member, romantic partner or very close friend died as a result of accident, homicide, or suicide?
No
Yes
If yes, how old were you? 
How did this person die?

Relationship to person lost

In the year before this person died, how often did you see/have? contact with him/her

5. When you were a child or more recently, did anyone (parent, other family member, romantic partner, stranger or someone else) ever succeed in physically forcing you to have intercourse, or oral or anal sex against your wishes or when you were in some way helpless?

No Yes If yes, at what age?

If yes, how many times? 1 , $2-4$ , 5-10 more than 10

If repeated, over what period? 6 mo. or less than 2 yrs. but less than 5 yrs.

7 mos. -2 yrs. , more

Who did this? (Specify stranger, parent, etc.)

Has anyone else ever done this to you? No Yes

6. Other than experiences described in item 5 , has anyone ever used physical force or threat to $T R Y$ to make you have intercourse, oral or anal sex, against your wishes or when you were in some way helpless?

No Yes If yes, at what age?

If yes, how many times? 1 $2-4$ 5-10 more than 10

If repeated, over what period? 6 mo. or less than 2 yrs. but less than 5 yrs.

7 mos. -2 yrs. more

Who did this? (Specify stranger, parent, etc.)

Has anyone else ever done this to you? No Yes

7. Other than experiences mentioned in items 5-6, has anyone ever actually touched private parts of your body or made you touch theirs against your wishes, or when you were in some way helpless?

No

Yes

If yes, at what age?

If yes, how many times? 1 $2-4$ , 5-10 more than 10

If repeated, over what period? $6 \mathrm{mo}$. or less than 2 yrs. but less than 5 yrs. 7 mos. -2 yrs. more

Who did this? (Specify sibling, date, etc.)

What age was this person?

Has anyone else ever done this to you? No Yes

8. When you were a child, did a parent, caregiver or other person ever slap you repeatedly, beat or otherwise attack or harm you?

No Yes If yes, at what age

If yes, how many times? 1 , 2-4 5-10 more than 10

If repeated, over what period? 6 mo. or less __, 7 mos.- 2 yrs. than 2 yrs. but less than 5 yrs 5 yrs. or more , more 
Describe force used against you (e.g., fist, belt)

Were you ever injured? If yes, describe

Who did this? (relationship to you)

Has anyone else ever done this to you? No Yes

9. Other than the experiences mentioned in item 8, have you ever been kicked, beaten, slapped around or otherwise physically harmed by a romantic partner, date, sibling, family member, stranger or someone else?

No Yes

If yes, at what age?

If yes, how many times? 1 $2-4$ $5-10$ more than 10

If repeated, over what period? 6 mo. or less than 2 yrs. but less than 5 yrs.

, 7 mos.- 2 yrs 5 yrs. or more

Describe force used against you (e.g., fist, belt)

Were you ever injured? If yes, describe

Who did this? (relationship to you)

If sibling, what age was he/she

Has anyone else ever done this to you? No Yes

10. Other than the experiences already covered, has anyone ever threatened you with a weapon like a knife or gun?

No Yes

If yes, how many times? 1 , $2-4$ more than 10

If repeated, over what period? 6 mo. or less than 2 yrs. but less than 5 yrs.

7 mos.- 2 yrs. 5 yrs. or more

If yes, at what age?

Describe nature of threat

Who did this? (relationship to you)

Has anyone else ever done this to you? No Yes

11. Have you ever been present when another person was killed, seriously injured, or sexually or physically assaulted?

$$
\text { No __ Yes }
$$

If yes, at what age?

Please describe what you witnessed

Was your own life in danger?

12. Have you ever been in any other situation where you were seriously injured or your life was in danger (e.g., involved in military combat or living in a war zone)?

No Yes

If yes, at what age?

Please describe. 
13. Have you ever been in any other situation that was extremely frightening or horrifying that has not been covered above?

No Yes

If yes, at what age? Please describe.

14. IF ANY OF THE EVENTS (OR ONGOING SITUATIONS) ALREADY DESCRIBED HAPPENED TO YOU MORE THAN ONCE, (e.g., TWO ROBBERIES, TWO DIFFERENT PEOPLE COMMITTING THE SAME ACT), PLEASE USE THE SPACE BELOW TO DESCRIBE EACH ADDITIONAL EVENT OR ONGOING SITUATION. PLEASE PROVIDE ALL INFORMATION REQUESTED UNDER THE ORIGINAL ITEM.

Item number Description.

\begin{tabular}{ll}
\hline & \\
\hline Item number $\_$Description. & \\
\hline & \\
\hline Item number & Description.
\end{tabular}

15. AS YOU FILLED OUT THIS QUESTIONNAIRE, DID YOU REPORT THE SAME INCIDENT, OR ONGOING SITUATION, UNDER MORE THAN ONE ITEM?

NO YES

IF YES, PLEASE INDICATE WHICH ITEMS REFER TO THE SAME INCIDENT

\section{References}

American Psychiatric Association (1994). Diagnostic and statistical manual of mental disorders (4th Ed.). Washington D.C.: Author.

Briere, J., \& Conte, J. (1993). Self-reported amnesia for abuse in adults molested as children. Journal of Traumatic Stress, 6, 21-31.

Briere, J., Elliott, D. M., Harris, K. \& Cotman, A. (1995). The Trauma Symptom Inventory: Psychometrics and association with childhood and adult victimization in clinical samples. Journal of Interpersonal Violence, 10, 387-401.

Foa, E., Riggs, D., Dancu, C., \& Rothbaum, B. (1993). Reliability and validity of a brief instrument for assessing posttraumatic stress disorder. Journal of Traumatic Stress, 6, 459-474.

Follette, V. M., Polusny, M., Bechtle, A. E., \& Naugle, A. E. (1996). Cumulative trauma: The impact of child sexual abuse, adult sexual assault, and spouse abuse. Journal of Traumatic Stress, 9, 25-35.

Goodman, L. A., Dutton, M. A., \& Harris, M. (1997). The relationship between violence dimensions and symptom severity among episodically homeless, mentally ill women. Joumal of Traumatic Stress, 10, 51-70.

Green, B. L. (1993). Identifying survivors at risk: Trauma and stressors across events. In J. P. Wilson \& B. Raphael (Eds.), International handbook of traumatic stress syndromes. New York: Plenum. 
Green, B. L. (1996). Trauma History Questionnaire (Self-report). In B.H. Stamm (Ed.), Measurement of stress, trauma, and adaptation (pp. 366-368). Lutherville, MD: Sidran.

Hammarberg, M. (1992). Penn Inventory for Posttraumatic Stress Disorder: Psychometric properties. Psychological Assessment, 4, 67-76.

Kessler, R. C., Sonnega, A., Bromet, E., Hughes, M., \& Nelson, C. (1995). Posttraumatic stress disorder in the national comorbidity survey. Archives of General Psychiatry, 52, 1048-1060.

Koss, M. P., Gidycz, C. A., \& Wisniewski (1987). Journal of Consulting and Clinical Psychology, $2,162-170$.

Koss, M. P., \& Oros, C. J. (1982). The Sexual Experiences Survey: A research instrument investigating sexual aggression and victimization. Journal of Consulting and Clinical Psychology, 50, 455-457.

Krinsley, K. E., \& Weathers, F. W. (1995). The assessment of trauma in adults. The National Center for Post-Traumatic Stress Disorder PTSD Research Quarterly, 6, 3, 1-6.

Krinsley, K. E., Gallagher, J. G., \& Weathers, F. W. (June, 1996). The development of a trauma history protocol: Defining and assessing reliability and validity. Paper presented at the International Society for Traumatic Stress Studies Second World Conference, Jerusalem, Israel.

Kubany, E. (1995). The Traumatic Life Events Questionnaire (TLEQ): A brief measure of prior trauma exposure. Unpublished scale. Available from the author.

Lauterbach, D., \& Vrana, S. (1996). Three studies on the reliability and validity of a self-report measure of posttraumatic stress disorder. Manuscript submitted for publication.

Norris, F. H. (1990). Screening for traumatic stress: A scale for use in the general population. Journal of Applied Social Psychology, 20, 1704-1718.

Norris, F. H. (1992). Epidemiology of trauma: Frequency and impact of different potentially traumatic events on different demographic groups. Journal of Consulting and Clinical Psychology, 60, 409-418.

Norris, F. H., \& Perilla, J. L. (1996). The Revised Civilian Mississippi Scale for PTSD: Reliability, validity, and cross-language stability. Journal of Traumatic Stress, 9, 285-298.

Norris, F. H., \& Riad, J. K. (1997). Standardized self-report measures of civilian trauma and posttraumatic stress disorder. In J. Wilson \& T. Keane (Eds.), Assessing psychological trauma and PTSD (pp. 7-42). New York: Guilford.

Resnick, H. S., Falsetti, S. A., Kilpatrick, D. G., \& Freedy, J. R. (1996). Assessment of rape and other civilian trauma-related PTSD: Emphasis on assessment of potentially traumatic events. In T. W. Miller (Ed.), Theory and assessment of stressful life events, (2nd ed.) (pp. 235-271). Madison, CT. International Universities Press, Inc.

Resnick, H. S., Kilpatrick, D. G., Dansky, B. S., Saunders, B. E., \& Best, C. L. (1993). Prevalence of civilian trauma and posttraumatic stress disorder in a representative national sample of women. Journal of Consulting and Clinical Psychology, 61, 984-991.

Russell, D. E. H. (1986). The secret trauma: Incest in the lives of girls and women. New York: Basic Books.

Spitzer, R. L., Williams, J. B. W., Gibbons, M., \& First, M. B. (1990). SCID: Users guide for the Structured Clinical Interview for DSM-III-R. Washington DC: American Psychiatric Press.

Straus, M. (1989). Measuring intra-family conflict and violence: The Conflict Tactics Scales. In M. Straus \& R. Gelles (Eds.), Physical violence in American families: Risk factors and adaptations to violence in 8145 families (pp. 29-47). New Brunswick, NJ: Transaction Books.

Vrana, S., \& Lauterbach, D. (1994). Prevalence of traumatic events and post-traumatic psychological symptoms in a nonclinical sample of college students. Joumal of Traumatic Stress, 7, 289-302.

Vreven, D., Gudanowski, D., King, L., \& King, D. (1995). The Civilian Version of the Mississippi PTSD Scale: A psychometric evaluation. Journal of Traumatic Stress, 8, 91-110.

Williams, L. M. (1995). Recovered memories of abuse in women with documented child sexual victimization histories. Journal of Traumatic Stress, 8, 4, 649-673. 
Copyright of Journal of Traumatic Stress is the property of Kluwer Academic Publishing and its content may not be copied or emailed to multiple sites or posted to a listserv without the copyright holder's express written permission. However, users may print, download, or email articles for individual use. 